\title{
An improved, low-cost, hydroponic system for growing Arabidopsis and other plant species under aseptic conditions
}

\author{
Fulgencio Alatorre-Cobos ${ }^{1,2}$, Carlos Calderón-Vázquez ${ }^{1,3}$, Enrique Ibarra-Laclette ${ }^{1,4}$, Lenin Yong-Villalobos ${ }^{1}$, \\ Claudia-Anahí Pérez-Torres ${ }^{1}$, Araceli Oropeza-Aburto ${ }^{1}$, Alfonso Méndez-Bravo ${ }^{1,4}$, Sandra-Isabel González-Morales ${ }^{1}$, \\ Dolores Gutiérrez-Alanís ${ }^{1}$, Alejandra Chacón-López ${ }^{1,5}$, Betsy-Anaid Peña-Ocaña ${ }^{1}$ and Luis Herrera-Estrella ${ }^{* *}$
}

\begin{abstract}
Background: Hydroponics is a plant growth system that provides a more precise control of growth media composition. Several hydroponic systems have been reported for Arabidopsis and other model plants. The ease of system set up, cost of the growth system and flexibility to characterize and harvest plant material are features continually improved in new hydroponic system reported.

Results: We developed a hydroponic culture system for Arabidopsis and other model plants. This low cost, proficient, and novel system is based on recyclable and sterilizable plastic containers, which are readily available from local suppliers. Our system allows a large-scale manipulation of seedlings. It adapts to different growing treatments and has an extended growth window until adult plants are established. The novel seed-holder also facilitates the transfer and harvest of seedlings. Here we report the use of our hydroponic system to analyze transcriptomic responses of Arabidopsis to nutriment availability and plant/pathogen interactions.

Conclusions: The efficiency and functionality of our proposed hydroponic system is demonstrated in nutrient deficiency and pathogenesis experiments. Hydroponically grown Arabidopsis seedlings under long-time inorganic phosphate (Pi) deficiency showed typical changes in root architecture and high expression of marker genes involved in signaling and Pi recycling. Genome-wide transcriptional analysis of gene expression of Arabidopsis roots depleted of Pi by short time periods indicates that genes related to general stress are up-regulated before those specific to Pi signaling and metabolism. Our hydroponic system also proved useful for conducting pathogenesis essays, revealing early transcriptional activation of pathogenesis-related genes.
\end{abstract}

Keywords: Hydroponics, Arabidopsis, Root, Phosphate starvation, Pathogenesis

\section{Background}

Standardization of growth conditions is an essential factor to obtain high reproducibility and significance in experimental plant biology. While lighting, humidity, and temperature are factors that can be effectively controlled by using plant growth chambers or rooms, media composition can be significantly altered by the physiochemical characteristics and elemental contaminants of different batches of gelling agents $[1,2]$.

\footnotetext{
* Correspondence: Iherrera@langebio.cinvestav.mx

'Laboratorio Nacional de Genómica para la Biodiversidad (Langebio)/Unidad de Genómica Avanzada (UGA), Centro de Investigación y Estudios Avanzados del IPN, 36500 Irapuato, Guanajuato, México

Full list of author information is available at the end of the article
}

For example, the inventory of changes in root system architecture (RSA) as a plant adaptation to nutrient stress can be influenced by the presence of traces of nutrients in different brands or even batches of agar as reported for the Pi starvation response [1]. Detailed protocols for obtaining real nutrient-deficient solid media for several macro and micronutrients have been recently reported [1,2]. These protocols describe a careful selection of gelling agents based on a previous chemical characterization that increase the cost and time to set up experiments. In addition those problems associated with media composition, plant growth window is reduced in petri plates (maximum 2-3 weeks) [3]. In vitro culture time can be extended using glass jars 
but accessibility to the root system is then compromised. Furthermore, additional handling and thus unnecessary plant stress during seedlings transfer to new growth media as well as during plant material collection should be also considered when experiments on solid media are designed.

One strategy for circumventing all problems described above is the use of hydroponic systems for plant culture. Several hydroponic systems have been reported for Arabidopsis [4-13] and some of them are now commercially available (Aeroponics ${ }^{\odot}$ ) [12]. Most of these systems are integrated by a plastic, glass or polycarbonate container with a seed-holder constituted by rock wool, a polyurethane (sponge) piece, a steel or nylon mesh, polyethylene granulate, or a polyvinyl chloride (PVC) piece. Those are open systems, which allow axenic conditions or reduced algal contamination into liquid growth media but sterility is not possible.

Here, we describe step by step a protocol for setting up a simple and low-cost, hydroponic system that allows sterility conditions for growing Arabidopsis and other model plants. This new system is ideal for large-scale manipulation of seedlings and even for fully developed plants. Our system is an improved version of Schlesier et al. [8], in which the original glass jar and steel seed-holder are substituted by a translucent polypropylene (PE) container and a piece of high-density polyethylene (HDPE) mesh. All components are autoclavable, reusable, cheap, and readily available from local suppliers. The new device designed as seed-holder avoids the use of low-melting agarose as support for seeds, allowing a quick and easy transfer to new media conditions and/or harvest of plant material. The efficiency and functionality of our proposed system is demonstrated and exemplified in experiments that showed typical early transcriptional changes under $\mathrm{Pi}$ starvation and pathogen infection.

\section{Results and discussion}

\section{Description of the hydroponic system}

We have improved a previously reported hydroponic system, consisting of a glass jar and stainless piece integrated by a wire screen fixed between two flat rings and held in place by three legs [8], by a simpler and cheaper system assembled with a PE vessel and a seed-holder integrated by a circle-shape HDPE mesh and two PE rings (Figure 1A,B; Table 1). Vessels and mesh used here are readily available in local markets; vessels are actually food containers (Microgourmet ${ }^{\oplus}$, Solo Cup, USA, www. solocup.com) available in food package stores while the HDPE mesh is a piece of anti-aphid mesh acquired in local stores providing greenhouse supplies (www.textile sagricolas.mx). A small cotton plug-filled orifice in the container lid allows gas exchange to the system (Figure 1C). This ventilation filter reduces but does not eliminate high

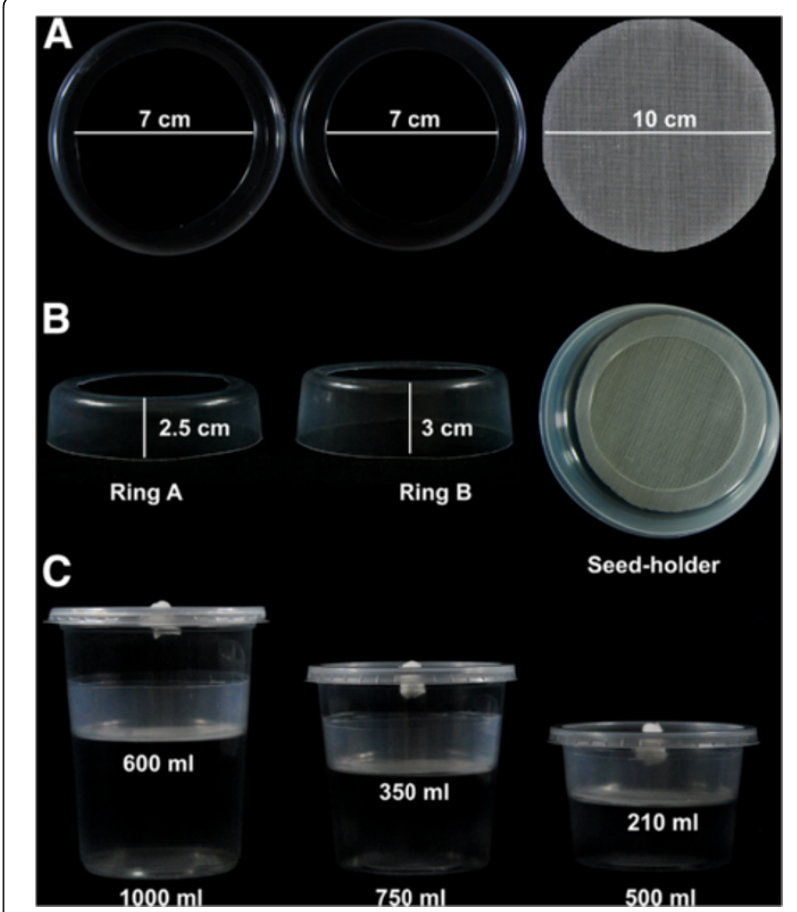

Figure 1 Hydroponic system: component dimensions and assembly. A and B) Dimensions and assembly of seed-holder. C) Assembled hydroponic system. Containers with different volume for liquid media are shown. The numbers at the bottom's container indicate the maximum volume and the number inside the container the volume of liquid media used in each case.

humidity in the medium container. Such problem could be solved adding more ventilation filters or using other sealing materials as micropore $3 \mathrm{M}^{\bullet}$ paper tape. Aeration of the liquid medium is not required for our hydroponic system. No negative effects on plant growth have been observed when small tanks are used as medium containers (references in Table 1).

The new seed-holder for positioning seeds on top of the liquid medium consists of a mesh of HDPE monofilaments held between two PE rings (ring A and B), with an area of $78.54 \mathrm{~cm}^{2}$ (diameter $=10 \mathrm{~cm}$ ) which is able to hold 50 to 65 Arabidopsis seedlings for up to 10-15 days after germination (Figure 1A,B; Figure 2) (Table 1). Fully developed Arabidopsis plants (2-3 plants per vessel) can also be grown in this system if the container lid is replaced by another PE container (Additional file 1). Anti-aphid mesh with a $0.75 \mathrm{~mm}$ by $0.75 \mathrm{~mm}$ opening size (mesh usually named $25 \times 25$ ) is adequate for keeping Arabidopsis seeds on top of the mesh (Figure 2A,B) and allowing independent root system development (Figure 2C,D,E). Antiaphid or anti-insect mesh with lower density can be useful for seeds larger than Arabidopsis seeds. No legs for supporting the mesh-holder are needed in our hydroponic system. The seed-holder is just placed into the container 
Table 1 Comparison between hydroponic systems previously reported and the system proposed here

\begin{tabular}{|c|c|c|c|c|c|c|}
\hline Parameter & $\begin{array}{l}\text { Agar-filled } \\
\text { plastic holder }\end{array}$ & $\begin{array}{l}\text { Rockwool-filled } \\
\text { plastic holder }\end{array}$ & $\begin{array}{c}\text { Sponge into a } \\
\text { polypropylene sheet }\end{array}$ & Polyethylene granulate & $\begin{array}{l}\text { Stainless mesh fixed two } \\
\text { metal rigs/Nylon mesh on } \\
\text { photo slide mount }\end{array}$ & This system \\
\hline Liquid medium container & Plastic box & Plastic box & Magenta GA-7 vessel ${ }^{\oplus}$ & Glass vessel & Round-rim glass jars/glass vessel & Plastic container \\
\hline Costs & Intermediate to high & Intermediate & High & High & High & Low \\
\hline Setup time & Intermediate to high & Intermediate & Low & Low & High & Low to intermediate \\
\hline Reuse of seed-holder & No & No & No & No & Yes/No & Yes \\
\hline Throughput & Intermediate & Intermediate & High & High & High/intermediate & Intermediate \\
\hline Container volume & Small to high & Small to intermediate & Small & Small to high & Intermediate & Intermediate to high \\
\hline Medium evaporation & High & High & Low & High & Low/High & Low \\
\hline Seedling number per holder & One & One & One & Many & Many & Many \\
\hline Sterility & No & No & Yes & No & Yes/No & Yes \\
\hline Aeration & Yes/No & Yes/No & No & No & No & No \\
\hline $\begin{array}{l}\text { Time for moving and sampling large } \\
\text { batches of plants between media }\end{array}$ & High & High & High & High & High & Low \\
\hline Development window & Adult plants & Adult plants & Seedling to adult plants & Seedling & Seedling & Seedling to adult plants \\
\hline References & {$[3,9,12]$} & {$[4,10,11]$} & [6] & [7] & {$[5,8,13]$} & \\
\hline
\end{tabular}




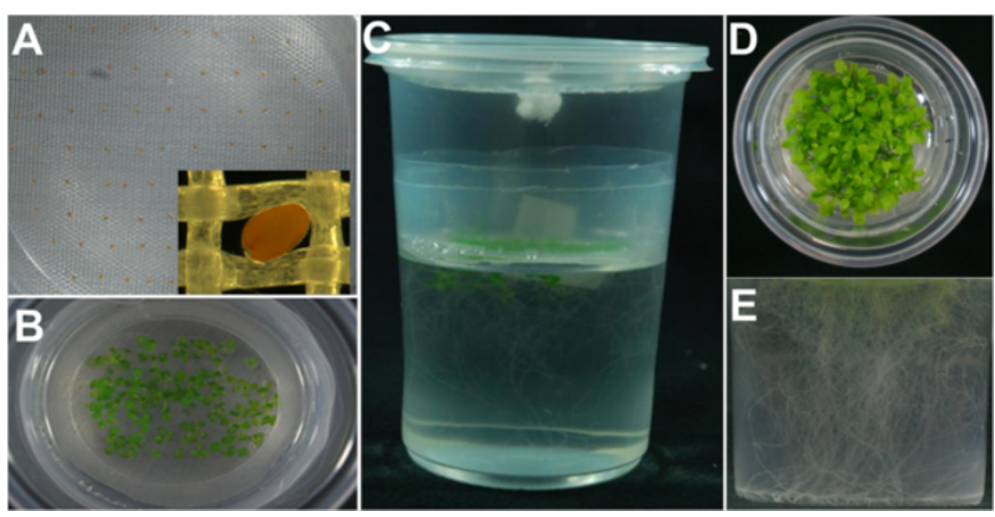

Figure 2 Arabidopsis seedlings growing under the hydroponic system proposed. A) Seeds sown on the mesh's seed-holder. A close-up view of a single seed is shown (inset). B-E) Seedlings growing in our hydroponic system. Top (B) and lateral view (C) of 12-day-old seedlings. Top (D) and lateral view (E) of 3-weeks-old seedlings.

and kept in place by pressing against the container walls. Unlike other protocols previously reported (Table 1), the container size of the system described here can vary according to volume of medium required (Figure 1C). However, the same standard seed-holder can be used for $1000 \mathrm{ml}, 750 \mathrm{ml}$, or $500 \mathrm{ml}$ containers, giving an effective volume for root growth of $600 \mathrm{ml}, 350 \mathrm{ml}$ and $210 \mathrm{ml}$, respectively (Figure 1C).

Our hydroponic system can be used for growing other model species under aseptic conditions. Solanum lycopersicum, Nicotiana tabacum, and Setaria viridis seeds were sterilized and directly sowed on the mesh. For all species, an adequate growth of shoot and root system was observed two weeks after germination (Figure 3).
Other advantages of this hydroponic system are related to plant transfer and plant tissue collection. For both, only a dressing tissue forceps (6 or 12 inch), previously sterilized, is required to pull up the seed-holder, and place it into new media (Figure 4A) or to submerge it into a liquid nitrogen container for tissue harvest (Figure 4B). Root harvest of young seedlings of the hydroponic system is also easier and less time-consuming than those from seedlings grown in agar media. When the seed-holder is taken out from the container, young roots adhere to mesh and can be blotted with an absorbent paper towel and immediately frozen in liquid nitrogen. Shoot biomass can be also easily detached from the mesh using a scalpel and then the mesh with the attached roots can be processed separately.

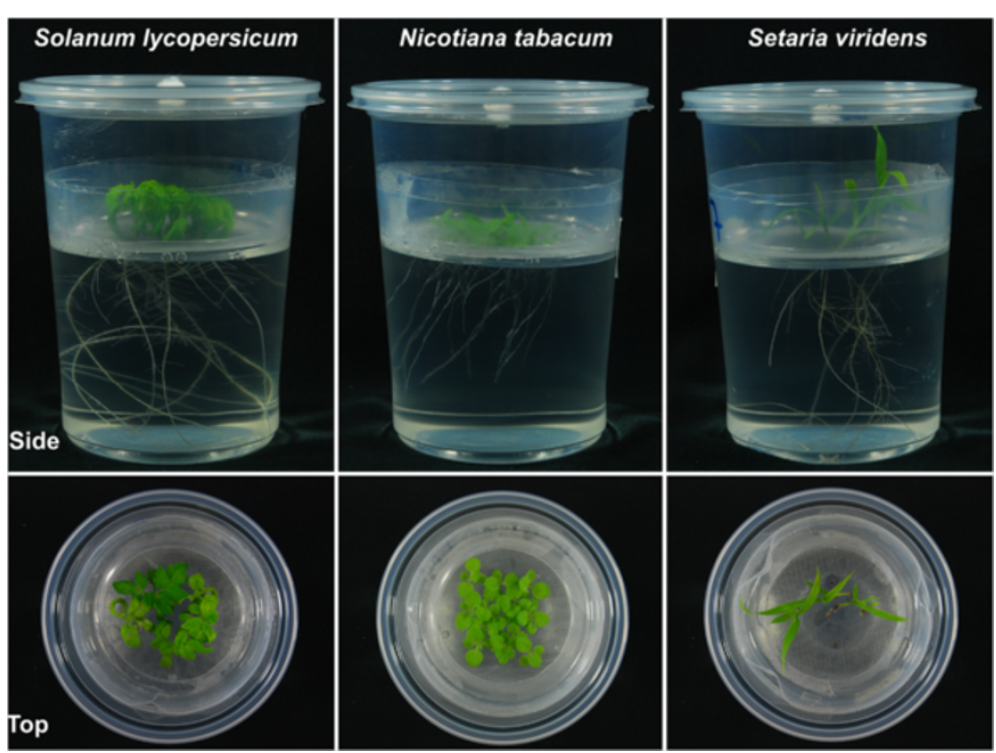

Figure 3 The hydroponic system proposed can be used with other model monocot and dicot plants. Lateral and top views of root and shoot growth of S. Iycopersicum, N. tabacum, and S. viridis at 2 to 3 weeks old. 


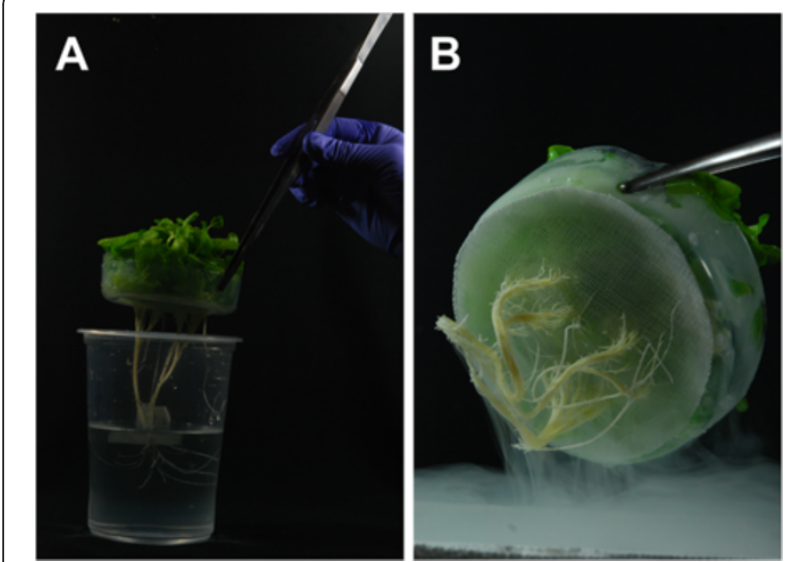

Figure 4 An easy and quick transfer to new growth media and/ or root harvesting can be carried out with this hydroponics system. A) Tobacco seedlings are transferred handling the seed-holder only. B) Batch of tobacco seedlings growing on the seed-holder frozen into liquid nitrogen

\section{Protocol for setting up the hydroponic system}

Step by step instructions for set up of hydroponic system are indicated in the following section and the Additional file 2. Tips and important notes are also indicated.

1. Getting a nylon mesh (See Figure 1A)

Get a piece of anti-aphid or anti-insect mesh. Draw a circle (10 $\mathrm{cm}$ diameter) using a marker and a cardboard template. Trim the circle using a fork. After tripping the circle, remove color traces on mesh using absolute alcohol. Wash the mesh under running water (Option: Use deionized water). Dry on paper towels. Tip: Use a red color marker for drawing. Red color is easier to clean than other colors.

2. Making a mesh holder (See Figure 1A,B)

Cut the $500 \mathrm{ml}$ PE container's bottom. Use a scalpel blade. Leave a small edge $(0.5 \mathrm{~cm}$ width). The mesh circle will put on this edge. For ring A, leave a height of $2.5 \mathrm{~cm}$, for ring $B$ leave $3 \mathrm{~cm}$. Tip: Use a scalpel blade with straight tip to cut easily the container's bottom.

3. Preparing the container lid

Locate the center of container lid and mark it. Drill the lid center. Seal the small lid hole with a cotton plug. Tip: Use a hot nail to melt a hole in the lid to avoid burrs.

4. Sterilization

Container and rings and mesh have to be separately sterilized by autoclaving $\left(121^{\circ} \mathrm{C}\right.$ and 15 psi pressure by 20 minutes). Put container, ring, and mesh groups into poly-bags. For container and rings, close but not seal the poly-bags. If so, pressure variations during sterilization could damage them. Important point: Put the autoclave in liquid media mode. Tip: After sterilization, put poly-bags into another bag for reducing contamination risks.

5. Hydroponic system assembly (See Figure 1C)

Open the sterilized poly-bags containing containers, rings, meshes, and lids. Put a volume of previously sterilized liquid medium into the container. Tip: the use liquid media at room temperature reduces the steam condensate on container lid and walls. Take a ring $\mathrm{B}$ with a dressing tissue forceps and put it into the container just above the liquid media level. Put a mesh piece on the ring $B$, lift it slowly and then return it on the ring avoiding to form bubbles. Fit the ring $\mathrm{A}$ onto the mesh piece. Tip: If it is difficult to fit the ring A onto the mesh piece, warm the ring quickly using a Bunsen burner. Finally, close the container.

\section{Applications of our hydroponic system: 1) Quick transcriptional responses to $\mathrm{Pi}$ starvation}

Applications of this new hydroponic culture system for model plants were analyzed in this study. Changes during Pi starvation at the transcriptional level associated with the Arabidopsis RSA modifications have been previously described [13]. Here, first we compared the effects of Piavailability on RSA and the expression profiles of eight marker genes for Pi deficiency in Arabidopsis seedlings grown in hydroponics versus agar media. Then, taking advantage of the short time that is required with this new hydroponic system for transferring plants to different media, early transcriptional responses to Pi depletion were explored at the genome-wide level; such responses have not been previously evaluated.

\section{Arabidopsis growth and Pi-depletion responsive genes on Pi-starved hydroponic media}

Arabidopsis seeds were germinated and grown for 12 days in hydroponics or agar media containing high-Pi (1.25 mM) or low-Pi $(10 \mu \mathrm{M})$ concentrations as previously reported [14,15]. By day 12 after germination, a higher shoot and root biomass was produced by Arabidopsis seedlings grown in hydroponics than those grown in solid media (Figure $5 \mathrm{~A}, \mathrm{~B}$ ), which is consistent with previous comparisons between both methods for growing Arabidopsis [5]. The typical increase in root biomass accumulation under Pi stress was observed in seedlings grown in agar medium, however such change was not statistically significant (Figure 5B). In contrast, the dry weight of roots of seedlings grown in hydroponics under Pi stress was 2.25fold higher compared to that observed for Pi-sufficient seedlings (Figure 5B). This higher root growth under low$\mathrm{Pi}$ is a typical RSA change that allows an increase of Pi uptake under natural soil conditions [14]. Regarding RSA adaptation to low Pi availability, we also found a 30\% reduction in primary root length with respect to control 

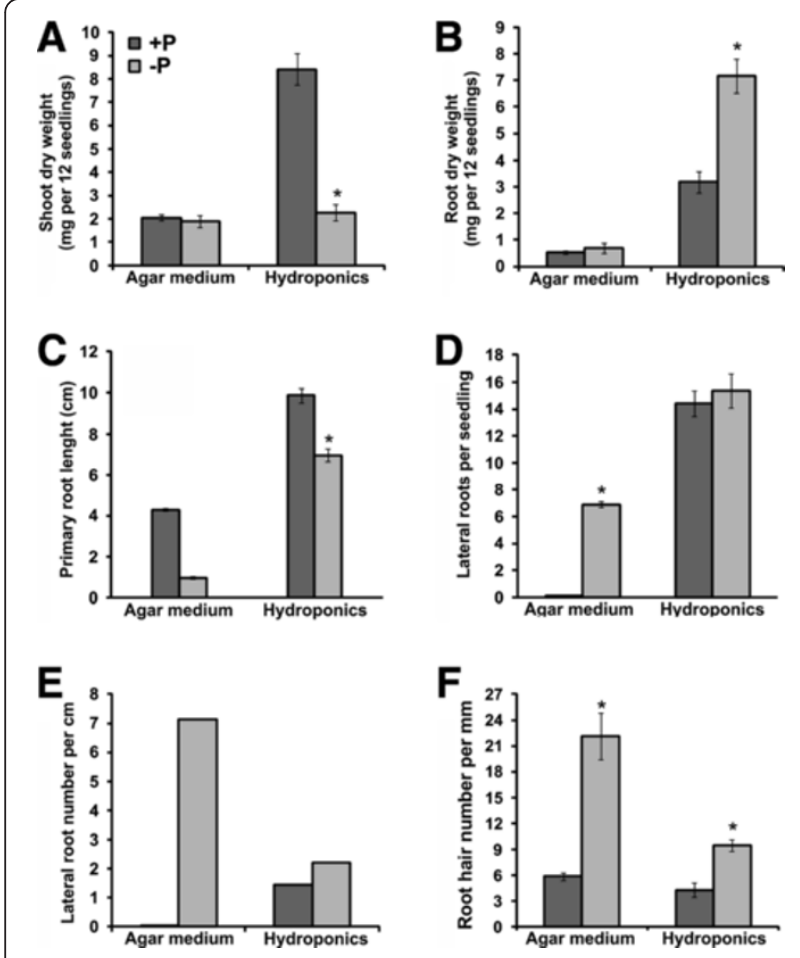

Figure 5 Plant growth under hydroponics or solid media under contrasting Pi regimens (A-F). Arabidopsis seedlings were directly sowed on the seed-holder (50 - 60 seed per mesh) or agar media (30-35 seeds per plate), growth for 12 days under two different $\mathrm{Pi}$ regimens $(-P=10 \mu \mathrm{MPi},+\mathrm{P}=1.25 \mathrm{mM} \mathrm{Pi})$ and then analyzed. Bars represent means $\pm S E$ (Hydroponics, biological replicates $=5, n=20-60$; agar medium, biological replicates $=10-15, n=15$ ). Asterisks denote a significant difference from corresponding control (+P treatment) according Student's t test $(P<0.05)$.

treatment under hydroponics while such reduction was higher $(76 \%)$ in roots from agar media (Figure 5C). Similarly, there was a modest increase in lateral root and root hair density under low-Pi in liquid media whereas a marked increase under same Pi growth condition was found in agar media (Figure 5D,E,F).

Although the effects of Pi deficiency on root development were more severe in agar media than in our hydroponic system, the typical root modifications induced by Pi stress (primary root shortening and higher production of lateral roots and root hairs) [14], were observed in both systems. Differences in the magnitude of RSA alterations in response to Pi-deprivation could be explained by variations in medium composition caused by gelling agents added, and/or the ease to access to Pi available in the growth systems used. It has been previously shown than contaminants such as $\mathrm{Pi}$, iron, and potassium in the gelling compounds can alter the morphophysiological and molecular response to Pi starvation [1]. Hydroponics provides a better control on media composition and allows a direct and homogenous contact of the whole root system with the liquid medium. This condition could be improve nutrient uptake, and under Pi starvation, alleviate the dramatic changes of RSA observed usually in roots of seedlings grown in agar media.

Afterwards, we determined the efficiency of the hydroponics system for inducing expression of low-Pi-responsive genes. Analysis of the expression profiles for eight genes involved with transcriptional, metabolic and morphological responses to Pi starvation were carried out in whole Arabidopsis seedlings that were grown in either low or high-Pi hydroponic conditions at 4, 7, 12, 14, 17 and 21 days. Transcript level quantification of the transcriptional factors (TF) PHR1 (PHOSPHATE STARVATION RESPONSE 1), WRKY75 (WRKY family TF) and bHLH32 (basic helixloop-helix domain-containing TF) revealed a direct influence of Pi stress persistence on the up-regulation of these three molecular modulators [16-18]. WRKY75 had the highest expression level among the TFs analyzed with a significant induction in expression after 12 days under $\mathrm{Pi}$ deficiency (Figure 6A). BHLH32 showed a similar increase in expression. As most molecular responses to Pi starvation are affected in phr1 mutant, PHR1 has been considered a

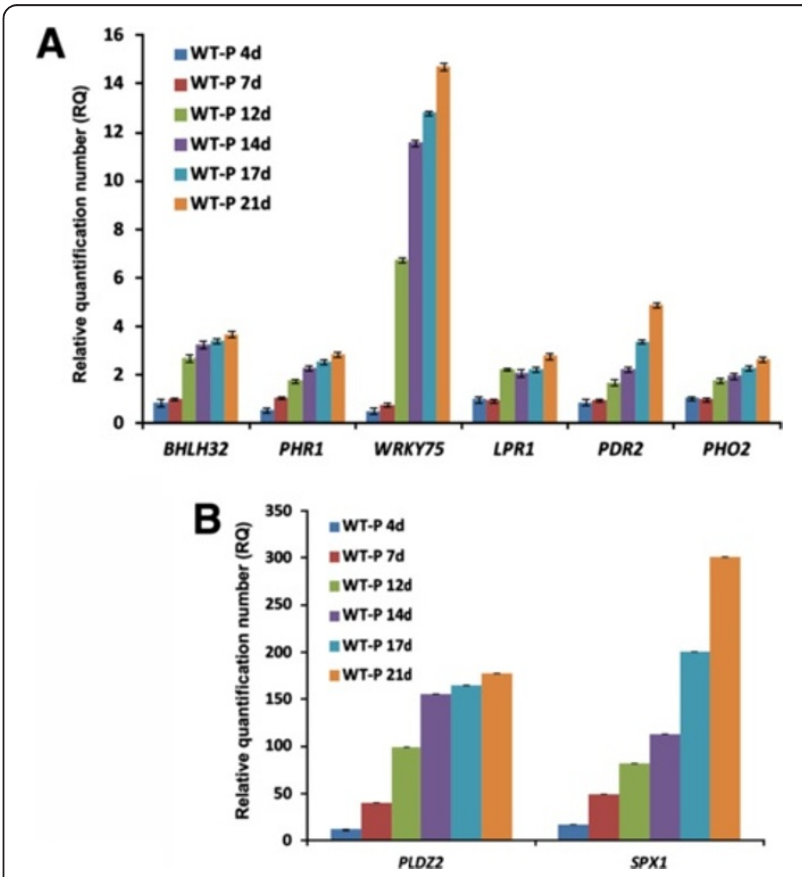

Figure 6 qRT-PCR expression profiling of marker genes for Pi starvation in Arabidopsis seedlings grown hydroponically. Expression profiling of $\mathbf{A}$ ) transcriptional modulators and genes involved with root meristem growth and B) Pi signaling and recycling. Arabidopsis seedlings were grown for 21 days under two different $\mathrm{Pi}$ regimens $(-P=10 \mu \mathrm{MPi},+P=1.25 \mathrm{mM} \mathrm{Pi})$. RNA of whole seedlings was extracted at six time points and gene expression levels were analyzed by qRT-PCR assays. Relative quantification number (RQ) was obtained from the equation $(1+E) 2^{\Delta C T}$ where $\Delta \Delta C T$ represents $\Delta C T(-P)-\Delta C T(+P)$, and $E$ is the $P C R$ efficiency. $C_{T}$ value was previously normalized using the expression levels of ACT2, PPR and UBHECT as internal reference. Data presented are means \pm SE of three biological replicates $(n=100-150)$. 
master controller of Pi signaling pathway $[16,19]$. In the case of PHR1 we found that this gene did not show as constitutive expression under $\mathrm{Pi}$ deficiency as originally reported [16]. Instead, the expression profile of this master regulator in roots showed responsiveness to low-Pi conditions (Figure 6A). These data are consistent with the low transcriptional induction of $P H R 1$ previously observed in Arabidopsis shoots [20]. LPR1 (LOW PHOSPHATE 1) and PDR2 (PHOSPHATE DEFICIENCY RESPONSE 2), two genes involved in root meristem growth [21], and the E2 ubiquitin conjugase PHOSPHATE 2 (PHO2/UBC24), related with Pi loading [22], showed a notable increase in expression after $14 \mathrm{~d}$ of treatment (Figure 6A). In contrast, SPX1 (a gene encoding a protein with a SYG1/Pho81/ XPHR1 domain) and PLDZ2 (PHOSPHOLIPASE DZ2), two typical marker genes of Pi deficiency implicated with Pi signaling and recycling [23,24] respectively, showed a significant induction starting at day four. Both SPX1 and PLDZ2, but especially $S P X 1$, had a marked increase in expression level (Figure 6B). The expression analysis of these Piresponsive genes together with RSA analyses during Pi starvation on hydroponics demonstrate the high performance of our system for plant growing and for analyzing molecular responses to nutrimental deficiency.

\section{Exploring early genome-wide transcriptional responses to Pi depletion: overview and functional classification of differentially expressed genes}

Early transcriptional responses to Pi availability at the genome-wide level ( $4 \mathrm{~h}$ to $<12 \mathrm{~h}$ ) have been previously determined in whole Arabidopsis seedlings using microarray platforms $[25,26]$. An important experimental condition in those studies has been the use of a 100-200 $\mu \mathrm{M}$ as a low-Pi concentration, considered enough to support biomass accumulation but not to induce an excessive Pi accumulation [26]. It has been reported that Arabidopsis seedlings growing at $100 \mu \mathrm{M} \mathrm{Pi}$ in agar media had similar endogenous phosphorus (P), biomass production and RSA to those growing at $1 \mathrm{mM} \mathrm{Pi}$ [14]. In liquid media, $200 \mu \mathrm{M}$ Pi has also been considered as a Pi-sufficient condition for growing monocot species such as maize [27]. We found that Arabidopsis seedlings grown with $150 \mu \mathrm{M}$ Pi in liquid media are not able to induce the expression of AtPT2/AtPHT1;4 (PHOSPHATE TRANSPORTER 2), a high-affinity Pi transporter responsive to Pi starvation reviewed in [28] as revealed by analysis of Arabidopsis seedlings harboring the transcriptional AtPT2::GUS reporter. Seedlings growing in hydroponics during 12 days showed null expression of the reporter in either shoot or root. When these seedlings were transferred to Pi-depleted media, AtPT2::GUS reporter was detected $12 \mathrm{~h}$ after transfer (Figure 7).

In order to demonstrate the efficiency of our system to elucidate early transcriptional responses, Arabidopsis seedlings were germinated and grown in the hydroponics system with $125 \mu \mathrm{M}$ Pi during 12 days, and then immediately deprived of Pi. Samples were taken at three short-time points (10 min, $30 \mathrm{~min}$, and $2 \mathrm{~h}$ ) (Figure $8 \mathrm{~A}$ ). Roots were harvested and frozen immediately after each time point, total RNA extracted and their transcriptome analyzed by microarray expression profiling. For data analyses, differences in gene expression between Pi-depleted versus $\mathrm{Pi}$-sufficient roots were identified (the overall $\mathrm{P}$ availability effect) and also the differences caused by the $\mathrm{Pi}$ availability by time interaction (time $\times \mathrm{Pi}$ effect). According to the stringency levels used (FDR $\leq 0.05$ and fold \pm 2 ), a total of 181 genes showed differential expression in at least one of three sampled time points (see Additional file 3). A total of 92 genes were found to be up-regulated and 89 down-regulated by Pi-depletion (Figure 8B). Interestingly, only 3 genes out of the 92 induced and 1 downregulated out of the 89 repressed were common to all three time points evaluated thus indicating specific transcriptional responses depending of the time point analyzed (Figure 8B). When clustered into functional classifications (Table 2 and Additional file 3), some resembled those previously reported [25-27,29], thus validating our system for high throughput transcriptional analyses. According to the expression profile, up-regulated genes were clustered in six different groups, whereas only three groups were identified for repressed genes (Additional file 3). Analysis of expression patterns by agglomerative hierarchical clustering showed a high number of upregulated genes in the last time point evaluated $(2 \mathrm{~h})$ while an opposite tendency was observed for down-regulated genes which were more responsive in the first time point (10 min) (Figure 8C). Differentially expressed genes were classified into functional categories according to The Munich Information Center for Protein Sequences classification (MIPS) using the FunCat database [30]. Categories more represented in up-regulated genes were those related with Metabolism, Transcription, Protein metabolism, and Interaction with the environment (Table 2). Also, there was a similar number of induced and repressed genes in $\mathrm{Pi}$, phospholipid, and phospholipid metabolism categories, with the exception of those related with glycolipid metabolism. Interestingly the Energy category (glycolysis, gluconeogenesis, pentose-phosphate pathway, respiration, energy conversion and regeneration, and light absorption) was only represented in induced genes (Table 2).

\section{Early transcriptional responses to low Pi availability involves cell wall modifications, protein activity,} oxidation-reduction processes, and hormones-mediated signaling that precede the reported Pi-signaling pathways According to the functional annotation of the Arabidopsis Information Resource database (TAIR, at www.arabidopsis. org), most genes, either induced or repressed during the 


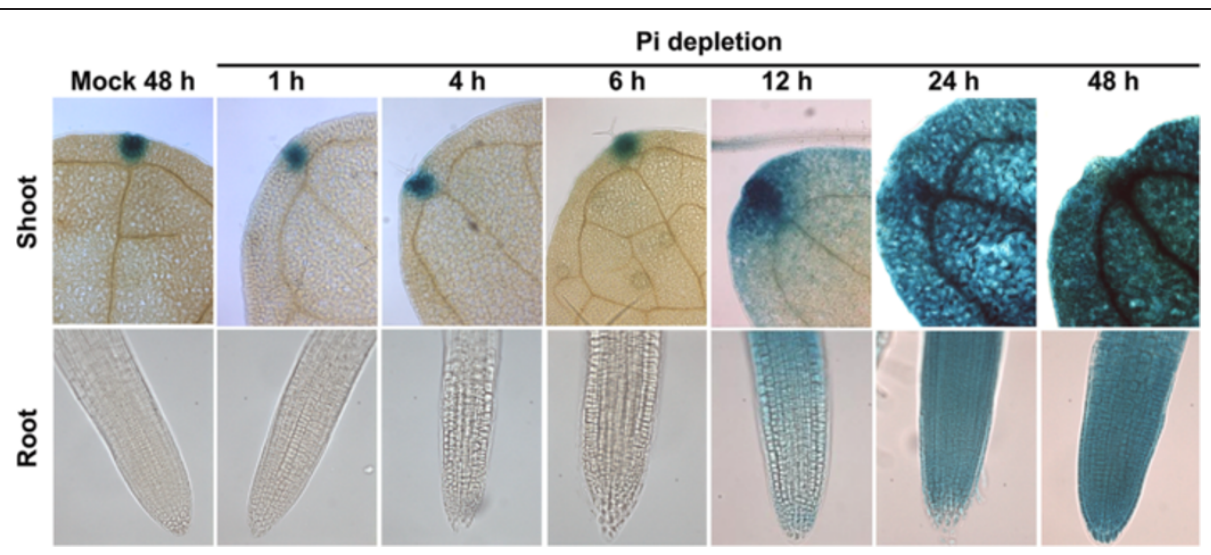

Figure 7 AtPT2::GUS expression pattern under Pi depletion. Arabidopsis AtPT2::GUS seedlings were grown hydroponically for 12 days under

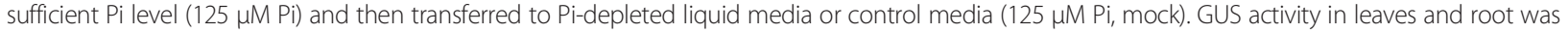
monitored by histochemical analyses at different time points. GUS expression in the mock condition is shown for the last time sampled (48 h).

A
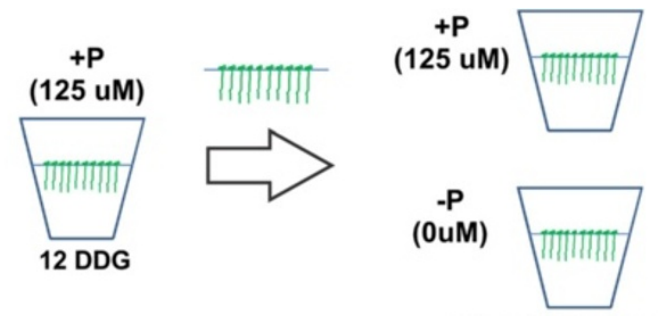

$10 \min , 30 \min , 2 \mathrm{~h}$

B
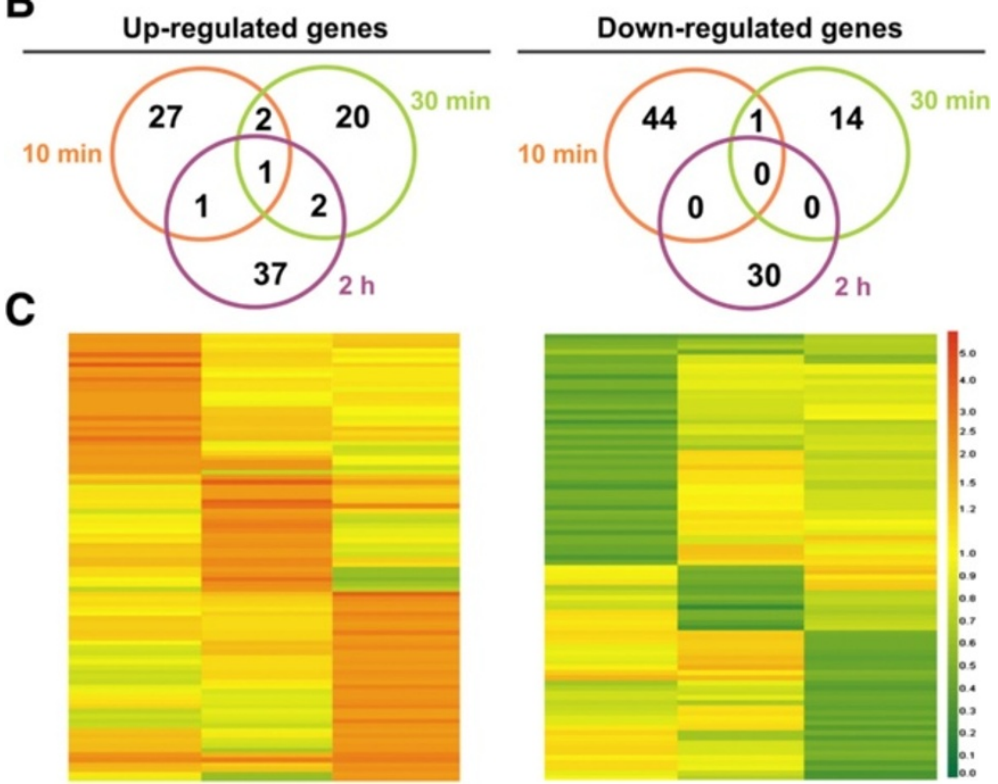

Figure 8 Early changes in the transcriptome of Arabidopsis roots under Pi starvation. A) Workflow for experiments. Arabidopsis seedlings were grown hydroponically for 12 days under sufficient Pi level (125 $\mu \mathrm{M} \mathrm{Pi}$ ) and then transferred to Pi-depleted liquid media for short times. Roots were harvested, RNA isolated and transcriptome analyzed using an oligonucleotide microarray platform. B) Edwards-Venn diagrams showing common or distinct regulated genes over the sampled time points. C) Clustering of differentially expressed genes. Clustering was performed using the Smooth correlation and average linkage clustering in GeneSpring GX 7.3.1 software (Agilent Technologies). Orange indicates up-regulated, green indicates down-regulated and white unchanged values, as shown on the color scale at the right side of the figure. 
Table 2 Distribution of functional categories of differentially expressed genes responding to Pi-deprivation under short time points in Arabidopsis roots

\begin{tabular}{|c|c|c|c|}
\hline \multirow[t]{3}{*}{ Functional category* } & \multirow{2}{*}{\multicolumn{3}{|c|}{$\begin{array}{l}\text { Up-/Down-regulated genes** }(\%) \\
\text { Time point sampled }\end{array}$}} \\
\hline & & & \\
\hline & $10 \mathrm{~min}$ & $30 \mathrm{~min}$ & $2 \mathrm{~h}$ \\
\hline Metabolism & $9.67 / 15.9$ & $21.4 / 7.14$ & $34.1 / 24.1$ \\
\hline Energy & $3.22 /-$ & 10.7/- & $2.43 /-$ \\
\hline Cell cycle and DNA processing & $3.22 / 2.27$ & $3.57 / 7.14$ & $4.87 / 6.89$ \\
\hline Transcription & $12.9 / 4.54$ & $7.14 / 21.4$ & $12.1 / 10.3$ \\
\hline Protein fate & $3.22 / 13.54$ & 7.14/7.14 & 12.1/13.7 \\
\hline Protein with binding function or cofactor requirement & $16.1 / 15.9$ & $32.1 / 7.14$ & $21.9 / 27.5$ \\
\hline Regulation of metabolism and protein function & - & $3.57 / 7.14$ & $2.43 / 3.44$ \\
\hline Cell transport, transport facilities, and transport routes & $9.67 / 4.54$ & $14.2 / 7.14$ & $7.31 / 10.3$ \\
\hline Cellular communication/signal transduction mechanism & $-/ 2.27$ & $-/ 14.2$ & $-/ 3.44$ \\
\hline Cell rescue, defense and virulence & $3.22 / 2.27$ & $10.7 / 14.2$ & $9.75 / 10.3$ \\
\hline Interaction with the environment & $9.67 / 2.27$ & $21.4 / 21.4$ & $2.43 / 6.89$ \\
\hline Systematic interaction with the environment & $3.22 / 2.27$ & $3.57 /-$ & $2.43 / 6.89$ \\
\hline Cell fate & $-/ 2.27$ & - & - \\
\hline Development & $6.45 / 2.27$ & $-/ 14.2$ & - \\
\hline Biogenesis of cellular components & - & $3.57 / 7.14$ & $2.43 / 6.89$ \\
\hline Subcellular localization & $29 / 34$ & $35.7 / 21.4$ & $26.8 / 34.4$ \\
\hline Unclassified proteins & $45.1 / 43.1$ & $21.4 / 35.7$ & $26.8 / 41.3$ \\
\hline
\end{tabular}

*Functional categories according to The Munich Information Center for Protein Sequences classification. ${ }^{* *}$ Differentially expressed genes with a fold change of at least \pm 2 at any time point and FDR $\leq 0.05$.

first 30 min of Pi depletion, are related to cell wall composition, protein activity, oxidation-reduction, and hormonesmediated signaling. Previously known Pi-responsive genes such MGDG SYNTHASE 3 (MGD3), SQDG SYNTHASE 2 (SQD2), PURPLE ACID PHOSPHATASE 22 (PAP22), and S-ADENOSYLMETHIONINE SYNTHASE 1 (SAM1) presented significant changes in expression until the last time point evaluated $(2 \mathrm{~h})$. Interestingly, a few transcriptional controllers were expressed differentially throughout the entire experiment.

At 10 minutes, Arabidopsis roots responded to Pideprivation with the activation of 27 genes (18.5\% of total) involved in polysaccharide degradation, callose deposition, pectin biosynthesis, cell expansion, and microtubule cytoskeleton organization (see group I, Additional file 3). Gene sets related with oxidation-reduction processes, protein activity modifications (ubiquitination, myristoylation, ATP or ion binding), and hormones-mediated signaling (abscisic acid, jasmonic acid) were also represented. Overrepresentation of groups according functional processes was not clear in down-regulated genes, excepting those related to modifications to protein fate (13.5\% of total 44 genes).

As Pi depletion progressed (30 $\mathrm{min})$, transcriptional changes related to cell wall decreased while responses to ion transport, signaling by hormones (auxins, abscisic acid, salicylic acid) or kinases were more represented in both induced and repressed genes (Additional file 3). In down-regulated genes, this trend was also found in the last time point $(2 \mathrm{~h})$. At 30 minutes, interestingly, genes involved with Pi-homeostasis, e.g. SPX1 and GLYCEROL3-PHOSPHATE PERMEASE 1 (G3Pp1), were already induced (see group IV and V, Additional file 3).

A higher number of up-regulated genes was found two hours after Pi-depletion. Most induced genes (9 out of 37 genes) were related to ion transport or homeostasis but also to carbohydrate metabolism, oxidation-reduction, signaling, protein activity and development. Importantly, other typical molecular markers for Pi starvation were also induced within 2 hours. Two phosphatidate phosphatases (PAPs) (At3g52820 and At5g44020) were induced gradually according Pi-starvation proceeded. MGD3 and SQD2, both involved with Pi recycling, were also induced at 2 hours (see group VI, Additional file 3). Expression of these genes, together with SPX1 and G3Pp1, indicate that the classical transduction pathways related with Pi-starvation can be triggered as early as two hours after seedlings are exposed to media lacking Pi. SPX1 is strongly induced by Pi starvation and usually classified as member of a system signaling pathway depending of SIZ1/PHR1 reviewed in [31]. Its early induction (3-12 h) has been previously reported [25] however an "immediate-early response" within few minutes after Pi depletion has been not reported so far. Likewise, a 
role for an enhanced expression of G3Pp1 inside transduction pathways or metabolic rearrangements triggered by Pi stress is still poorly understood [25,26]. A recent functional characterization of Arabidopsis glycerophosphodiester phosphodiesterase (GDPD) family suggests glycerol-3-phosphate (G3P) as source of Pi or phosphatidic acid (PA), which could be used by glycerol-3 phosphatase (GPP) or DGDG/SQDG pathways [32]. Early induced expressions of G3Pp1, PAP22, and MGD3 is in agreement with the hypothesis that under $\mathrm{Pi}$ deficiency G3P could be first converted into PA by two acyltransferase reactions and $\mathrm{Pi}$ would be then released during the subsequent conversion of PA into diacylglycerol (DAG) by PAPs [32]. DAG produced could be incorporated into DGDG or SQDG by MGD2/3 and DGD1/2 and SQD1/2, respectively [32]. MGD2 and MGD3 have been found induced in Arabidopsis seedlings depleted of Pi for 3-12 h [25]. This early transcriptional activity for $M G D$ genes during Pi starvation is also reflected in enhanced enzymatic activities as revealed in Pi-starved bean roots [33]. Increased PA levels and MGDG and DGDG activities have been reported in bean roots starved of Pi for less than $4 \mathrm{~h}$ [31]. Early gene expression activation of genes encoding MGDG and DGDG but not PLD/C enzymes suggests G3P and not PC as source for PA and DAG biosynthesis for early Pi signaling and recycling pathways.

According with our data, a specific transduction pathway to Pi deficiency could be preceded by general responses related to stress, which could modify metabolism before triggering specific expression of transcriptional factors. This idea is consistent with previous reports assaying Pi-depletion in Arabidopsis by short and medium-long times (3-48 h), which also reported differentially expressed genes related with pathogenesis, hormone-mediated signaling, protein activity, redox processes, ion transport, and cell wall modifications $[25,28,34]$. Similar results have been recently reported in rice seedlings under Pi starvation for $1 \mathrm{~h}$ [35].

\section{Applications of our hydroponic system: 2) Pathological assays to evaluate systemic defense responses}

In order to determine the suitability of our hydroponic system to perform Arabidopsis-pathogen interactions, we evaluated the systemic effect of root inoculation with Pseudomonas syringae pv tomato strain DC3000 (Pst DC3000) on transcriptional activation of the patho genesis-related gene $P R 1$. Although $P$. syringae is generally known as a leaf pathogen, it has been proven to be an excellent root colonizer in Arabidopsis [36,37]. Transgenic Arabidopsis seedlings carrying the PR1::GUS construct were grown for 12 days and then inoculated with $0.002 \mathrm{OD}_{600}$ of fresh bacterial inoculum. $\beta$-glucoronidase (GUS) activity was analyzed by histochemical staining at different time intervals after inoculation. Systemic response to Pst root colonization was evident between 2 and 6 hours after inoculation (hai), as revealed by strong expression of the marker gene in leaves. After 24 hai, GUS activity spread throughout the whole shoot system, but not in roots (Figure 9). These results demonstrate a good performance for studying plant responses to pathogens.

\section{Conclusions}

Here, we describe a practical and inexpensive hydroponic system for growing Arabidopsis and other plants under sterile conditions with an in vitro growth window that goes from seedlings to adult plants. Our system uses recyclable and plastic materials sterilizable by conventional autoclaving that are easy to get at local markets. In contrast to other hydroponic systems previously reported, the components of the system (container size, mesh density, lid) described here can be easily adapted to different experimental designs or plant species. The seed-holder avoids the use of an agarose plug or any other accessory reducing time for setting up experiments and decreasing risks of contamination.

Applications and advantages of our hydroponic system are exemplified in this report. First, rapid transcriptome changes of Arabidopsis roots induced by $\mathrm{Pi}$ depletion

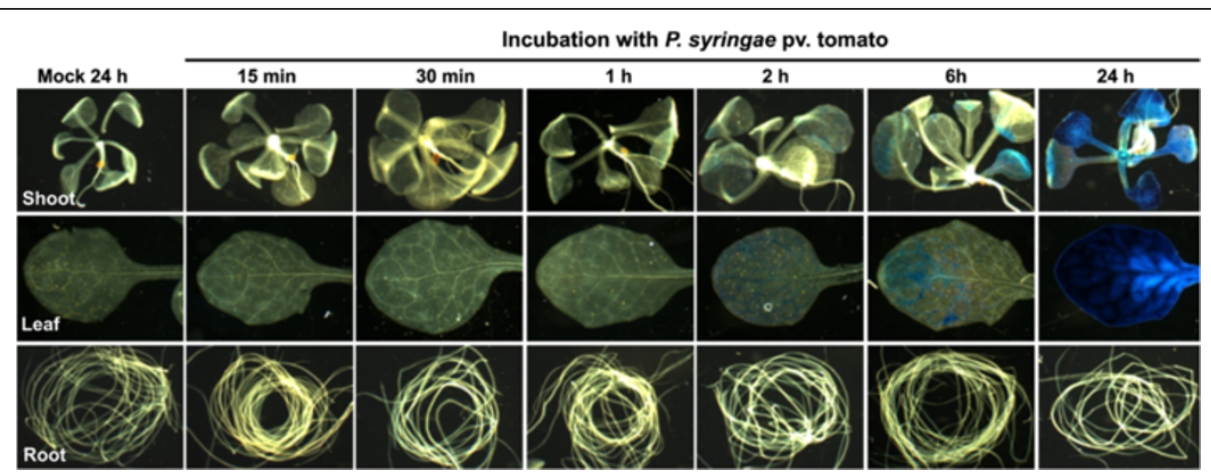

Figure 9 PR1::GUS expression pattern under P. syringae pv. tomato incubation. Arabidopsis PR1::GUS seedlings were grown hydroponically and then transferred to liquid media containing P. syringae bacteria (final $0.002 \mathrm{OD}_{600}$ ) or control media (mock). GUS activity in shoot and root was monitored by histochemical analyses at different time points. GUS expression in mock condition is shown for the last time point sampled (24 h). 
were detected by a rapid harvest from growth media using our new seed-holder designed. Our analyses confirm that Arabidopsis roots early responses to Pi depletion includes the activation of signaling pathways related to general stress before to trigger those specific to $\mathrm{Pi}$ stress, and support the idea that G3P could be a source of $\mathrm{Pi}$ and other molecules as PA during early signaling events induced by Pi starvation. Second, our hydroponic system showed a high performance to set up pathogenesis assays.

\section{Methods}

\section{Growth media}

For solid or liquid media, a $0.1 \mathrm{X}$ Murashige and Skoog (MS) medium, $\mathrm{pH} 5.7$, supplemented with $0.5 \%$ sucrose (Sigma-Aldrich), and $3.5 \mathrm{mM}$ MES (Sigma-Aldrich) was used. For solid growth media, agar plant TC micropropagation grade (composition/purity not provided) (A296, Phytotechnology Laboratories, US) from Gelidium species was used at $1 \%(\mathrm{~W} / \mathrm{V})$.

\section{Plant material and growth conditions}

Arabidopsis thaliana ecotype Columbia (Col-0, ABRC stock No. 6000 from SALK Institute), marker lines PR1:: GUS (At2g14610) (kindly provided by F.M. Ausubel) and AtPT2::GUS (At2g38940) [38], tobacco cv Xhanti, tomato cv Micro-Tom, and Setaria viridis seeds were used in this study. In all cases, seeds were surface sterilized by sequential treatments with absolute ethanol for $7 \mathrm{~min}, 20 \%$ (v/v) commercial bleach for $7 \mathrm{~min}$ and rinsed three times with sterile distilled water. Previous sterilization, Setaria seeds were placed at $-80^{\circ} \mathrm{C}$ overnight as recommended [39]. Arabidopsis sterilized seeds were vernalized at $4^{\circ} \mathrm{C}$ for 2 days (solid media) or 4 days (hydroponics). For Arabidopsis, 50-65 seeds were directly sowed on the mesh of the seed-holder, and 30-35 seeds in Petri plates (50 ml volume, $15 \mathrm{~mm} \times 150 \mathrm{~mm}$, Phoenix Biomedical). Seedlings of all species evaluated were grown at $22^{\circ} \mathrm{C}$, except tobacco which was grown at $28^{\circ} \mathrm{C}$, using growth chambers (Percival, Perry, IA, USA) with fluorescent light $\left(100 \mu \mathrm{mol} \mathrm{m} \mathrm{m}^{-2} \mathrm{~s}^{-1}\right)$ and a photoperiod of $16 \mathrm{~h}$ light $/ 8 \mathrm{~h}$ dark.

\section{Analysis of root architecture traits}

To determine root architecture traits, seedlings were grown on agar plates at an angle of $65^{\circ}$ or under hydroponic conditions. Root length was measured from root tip to hypocotyls base. For lateral root (LR) quantification, all clearly visible emerged secondary roots were taken into account when the number of LRs was determined. Root hair density was calculated from root images taken with a digital camera connected to an AFX-II-A stereomicroscope (Nixon, Tokyo). Statistical analysis of quantitative data was performed using the statistical tools (Student's $t$ test) of Microsoft Excel software.

\section{GUS analyses}

For histochemical analysis of GUS activity, Arabidopsis seedlings were incubated for $4 \mathrm{~h}$ at $37^{\circ} \mathrm{C}$ in a GUS reaction buffer $\left(0.5 \mathrm{mg} \mathrm{ml}^{-1}\right.$ of 5-bromo-4-chloro-3-indolylb-D-glucuronide in $100 \mathrm{mM}$ sodium phosphate buffer, $\mathrm{pH}$ 7). Seedlings were cleared using the method previously described [40]. At least 15 transgenic plants were analyzed and imaged using Normarski optics on a Leica DMR microscope.

\section{Microarray analysis}

Roots were collected and immediately frozen and RNA isolated using the Trizol reagent (Invitrogen) and purified with the RNeasy kit (Qiagen) following the manufacturer's instructions. Spotten glass microarray slides (Arabidopsis Oligonucleotide Array version 3.0) were obtained from University of Arizona (http://.ag.arizona.edu/ microarray/). Three biological replicates (150 seedlings per replicate) were used for RNA isolation and two technical replicates (in swap) to the two channel microarrays. Fluorescent labeling of probes, slide hybridization, washing, and image processing was performed as described [27]. A loop design was used in order to contrast the gene expression differences between treatments and time points. Microarray normalization and data analysis to identify differentially expressed genes with at least two-fold change in expression were carried as previously reported [27].

The microarray data have been deposited in Gene Expression Omnibus (GEO) and are accessible through GEO Series, accession number: GSE53114 (http://www.ncbi. nlm.nih.gov/geo/query/acc.cgi?acc=GSE53114).

\section{Transcript analysis}

Total RNA was extracted with Trizol (Invitrogen) and purified using Qiagen RNeasy columns according to the manufacturer's instructions. cDNA was synthesized using $30 \mu \mathrm{g}$ of total RNA with SuperScript III Reverse Transcriptase (Invitrogen) and used for qRT-PCR (7500 Real Time PCR System, Applied Biosystems). Expression of marker genes for Pi starvation was analyzed using the oligonucleotides listed in the Additional file 4. Gene expression analyses were performed as previously reported [27]. Briefly, Relative quantification number (RQ) was obtained from the equation $(1+\mathrm{E}) 2^{\Delta \Delta \mathrm{C}}$ where $\Delta \Delta \mathrm{CT}$ represents $\Delta \mathrm{CT}$ (Treatment)- $\Delta \mathrm{CT}$ (Control) and $\mathrm{E}$ is the PCR efficiency. Each CT was previously normalized using the expression levels of ACT2 (At3g18780), PPR (At5g55840), and UPL7 (At3g53090) as internal references.

\section{Pseudomonas syringae bioassays}

Bacterial strain P. syringae pv tomato (Pst) DC3000 was cultured on King's B medium (KB) supplied with $50 \mu \mathrm{g} \mathrm{ml}{ }^{-1}$ rifampicin. PR1::GUS seedlings were grown hydroponically as described above. For infection of 12 day-old seedlings, a 
bacterial culture was grown overnight in $\mathrm{KB}$ at $28^{\circ} \mathrm{C}$. Bacteria were centrifuged, washed three times with sterilized water and resuspended in water to a final $\mathrm{OD}_{600}$ of 0.04 . The appropriate volume was added to seedling medium to a final $\mathrm{OD}_{600}$ of 0.002 . After infection, plant material was harvested at several time points for GUS histochemical assays and analyzed under a Leica DMR microscope.

\section{Additional files}

Additional file 1: 35-40-day-old Arabidopsis plants growing under our hydroponic system proposed. The Arabidopsis seeds were directly sowed on the seed-holder and three adult plants per vessel were grown until the flowering began.

Additional file 2: Video showing the assembly process for our hydroponic system.

Additional file 3: Differentially expressed genes by Pi depletion during short time points in Arabidopsis roots.

Additional file 4: qRT-PCR primers used in this study.

\section{Abbreviations}

MGDG: Monogalactosyldiacylglycerol; DGDG: Digalactosyldiacylglycerol; SQDG: Sulfoquinovosyldiacylglycerol.

\section{Competing interests}

The authors declare that they have no competing interests.

\section{Authors' contributions}

FAC, CCV and LHE conceived the new hydroponic system and designed the experiments. FAC, CCV, EIL, LYV, C-APT, AOA, AMB, S-IGM, DAG, and B-APO performed the experiments. FAC, CCV, EIL, and LHE analyzed the data. FAC, $C C V$, and LHE drafted the manuscript. All authors read and approved the final manuscript.

\section{Acknowledgements \\ This work was partially funded by the Howard Medical Institute (Grant 55003677) to LHE. FAC is indebted to CONACYT for a PhD fellowship (190577). The authors would like to thank R. Sawers (Langebio) for providing S. viridens seeds, J. H. Valenzuela for $P$. syringae pv tomato strain, J. Antonio Cisneros-Durán (Cinvestav Unidad Irapuato) for his great help with photog- raphy and video in this work, and Flor Zamudio-Hernández and María de J. Ortega-Estrada for microarray and qRT-PCR services. We are grateful to the anonymous reviewers for their positive comments and meticulous revision for improving the quality of this manuscript.}

\section{Author details \\ ${ }^{1}$ Laboratorio Nacional de Genómica para la Biodiversidad (Langebio)/Unidad de Genómica Avanzada (UGA), Centro de Investigación y Estudios Avanzados del IPN, 36500 Irapuato, Guanajuato, México. ${ }^{2}$ Current address: Department of Biological and Environmental Sciences, Institute of Biotechnology, University of Helsinki, 00014 Helsinki, Finland. ${ }^{3}$ Current address: Instituto Politécnico Nacional, Centro Interdisciplinario de Investigación para el Desarrollo Integral Regional Unidad Sinaloa, 81101 Guasave, Sinaloa, México. ${ }^{4}$ Current address: Red de Estudios Moleculares Avanzados, Instituto de Ecología A.C. Carretera Antigua a Coatepec \#351, Xalapa 91070, Veracruz, México. ${ }^{5}$ Current address: Instituto Tecnológico de Tepic, Laboratorio de Investigación Integral en Alimentos, División de Estudios de Posgrado, 63175 Tepic, Nayarit, México.}

Received: 15 December 2013 Accepted: 13 March 2014

Published: 21 March 2014

\section{References}

1. Jain A, Poling MD, Smith AP, Nagarajan VK, Lahner B, Meagher RB, Raghothama KG: Variations in the composition of gelling agents affect morphophysiological and molecular responses to deficiencies of phosphate and other nutrients. Plant Physiol 2009, 150:1033-1049.

2. Gruber BD, Giehl RFH, Friedel S, von Wirén N: Plasticity of the Arabidopsis root system under nutrient deficiencies. Plant Physiol 2013, 163:161-179.

3. Conn SJ, Hocking B, Dayod M, Xu B, Athman A, Henderson S, Aukett L, Conn V, Shearer MK, Fuentes S, Tyerman SD, Gilliham M: Protocol: optimising hydroponic growth systems for nutritional and physiological analysis of Arabidopsis thaliana and other plants. Plant Methods 2013, 9:4. doi:10.1186/1746-4811-9-4.

4. Gibeaut DM, Hulett J, Cramer GR, Seemann JR: Maximal biomass of Arabidopsis thaliana using a simple, low-maintenance hydroponic method and favorable environmental conditions. Plant Physiol 1997, 115:317-319.

5. Toda T, Koyama H, Hara T: A simple hydroponic culture method for the development of a highly viable root system in Arabidopsis thaliana. Biosci Biotechnol Biochem 1999, 63:210-212.

6. Arteca RN, Arteca JM: A novel method for growing Arabidopsis thaliana plants hydroponically. Physiol Plant 2000, 108:188-193.

7. Battke F, Schramel P, Ernst D: A novel method for in vitro culture of plants: cultivation of barley in a floating hydroponic system. Plant Mol Biol Rep 2003, 21:405-409.

8. Schlesier B, Bréton F, Mock H-P: A hydroponic culture system for growing Arabidopsis thaliana plantlets under sterile conditions. Plant Mol Biol Rep 2003, 21:449-456.

9. Norén $\mathrm{H}$, Svensson $\mathrm{P}$, Andersson B: A convenient and versatile hydroponic cultivation system for Arabidopsis thaliana. Physiol Plant 2004, 121:343-348.

10. Robison MM, Smid MPL, Wolyn DJ: High-quality and homogeneous Arabidopsis thaliana plants from a simple and inexpensive method of hydroponic cultivation. Can J Bot 2006, 84:1009-1012.

11. Huttner D, Bar-Zvi D: An improved, simple, hydroponic method for growing Arabidopsis thaliana. Plant Mol Biol Rep 2003, 21:59-63.

12. Tocquin P, Corbesier L, Havelange A, Pieltain A, Kurtem E, Bernier G, Périlleux C: A novel high efficiency, low maintenance, hydroponic system for synchronous growth and flowering of Arabidopsis thaliana. BMC Plant Biol 2003, 3:2.

13. Bargmann BOR, Birnbaum KD: Fluorescence activated cell sorting of plant protoplasts. J Vis Exp 2010, 36. http://www.jove.com/details.php?id=1673, doi:10.3791/1673

14. López-Bucio J, Hernández-Abreu E, Sánchez-Calderón L, Nieto-Jacobo MF, Simpson J, Herrera-Estrella L: Phosphate availability alters architecture and causes changes in hormone sensitivity in the Arabidopsis root system. Plant Physiol 2002, 129:244-256.

15. Pérez-Torres CA, López-Bucio J, Cruz-Ramírez A, Ibarra-Laclette E, Dharmasiri S, Stelle M, Herrera-Estrella L: Phosphate availability alters lateral root development in Arabidopsis by modulating auxin sensitivity via a mechanism involving the TIR1 auxin receptor. Plant Cell 2008, 12:3258-3272.

16. Rubio V, Linhares F, Solano R, Martín AC, Iglesias J, Leyva A, Paz-Ares J: A conserved MYB transcription factor involved in phosphate starvation signaling both in vascular plants and in unicellular algae. Gene Dev 2001, 5:2122-2133.

17. Devaiah BN, Karthikeyan AS, Raghothama KG: WRKY75 transcription factor is a modulator of phosphate acquisition and root development in Arabidopsis. Plant Physiol 2007, 143:1789-17801.

18. Chen ZH, Nimmo GA, Jenkins Gl, Nimmo HG: BHLH32 modulates several biochemical and morphological processes that respond to Pi starvation in Arabidopsis. Biochem J 2007, 405:191-198.

19. Bustos R, Castrillo G, Linhares F, Puga Ml, Rubio V, Pérez-Pérez J, Solano R, Leyva A, Paz-Ares J: A central regulatory system largely controls transcriptional activation and repression responses to phosphate starvation in Arabidopsis. PLoS Genet 2010, 6:e1001102

20. Nilsson L, Müller R, Nielsen TH: Increased expression of the MYB-related transcription factor, PHR1, leads to enhanced phosphate uptake in Arabidopsis thaliana. Plant Cell Environ 2007, 30:1499-1512.

21. Ticconi CA, Lucero RD, Sakhonwasee S, Adamson AW, Creff A, Nussaume L, Desnos T, Abel S: ER-resident proteins PDR2 and LPR1 mediate the developmental response of root meristems to phosphate availability. Proc Natl Acad Sci U S A 2009, 106:14174-14179.

22. Aung K, Lin SI, Wu CC, Huang YT, Su CL, Chiou TJ: pho2, a phosphate overaccumulator, is caused by a nonsense mutation in a microRNA399 target gene. Plant Physiol 2006, 141:1000-1011.

23. Duan K, Yi K, Dang L, Huang H, Wu W, Wu P: Characterization of a sub-family of Arabidopsis genes with the SPX domain reveals their 
diverse functions in plant tolerance to phosphorus starvation. Plant $\mathrm{J}$ 2008, 54:965-975

24. Cruz-Ramírez A, Oropeza-Aburto A, Razo-Hernández F, Ramírez-Chávez E, Herrera-Estrella L: Phospholipase DZ2 plays an important role in extraplastidic galactolipid biosynthesis and phosphate recycling in Arabidopsis roots. Proc Natl Acad Sci U S A 2006, 103:6765-6770.

25. Misson J, Raghothama KG, Jain A, Jouhet J, Block MA, Bligny R, Ortet P, Creff A, Somerville S, Rolland N, Doumas P, Nacry P, Herrera-Estrella L, Nussaume L, Thibaud MC: A genome-wide transcriptional analysis using Arabidopsis thaliana Affymetrix gene chips determined plant responses to phosphate deprivation. Proc Natl Acad Sci U S A 2005, 102:1 1934-11939.

26. Morcuende R, Bari R, Gibon Y, Zheng W, Pant BD, Bläsing O, Usadel B, Czechowski T, Udvardi MK, Stitt M, Scheible WR: Genome-wide reprogramming of metabolism and regulatory networks of Arabidopsis in response to phosphorus. Plant Cell Environ 2007, 30:85-112.

27. Calderón-Vázquez C, Ibarra-Laclette E, Caballero-Pérez J, Herrera-Estrella L: Transcript profiling of Zea mays roots reveals gene responses to phosphate deficiency at the plant- and species-specific levels. J Exp Bot 2008, 59:2479-2497.

28. Nussaume L, Kanno S, Javot H, Marin E, Pochon N, Ayadi A, Nakanishi TM, Thibaud M-C: Phosphate import in plants: focus on the PHT1 transporters. Front Plant Sci 2011, 2:83.

29. Chacón-López A, Ibarra-Laclette E, Sánchez-Calderón L, Gutiérrez-Alanis D, Herrera-Estrella L: Global expression pattern comparison between low phosphorus insensitive 4 and WT Arabidopsis reveals an important role of reactive oxygen species and jasmonic acid in the root tip response. Plant Signal Behav 2011, 6(3):382-392.

30. Ruepp A, Zollner A, Maier D, Albermann K, Hani J, Mokrejs M, Tetko I, Güldener U, Mannhaupt G, Münsterkötter M, Mewes HW: The FunCat, a functional annotation scheme for systematic classification of proteins from whole genomes. Nucleic Acids Res 2004, 32:5539-5545.

31. Nilsson $L$, Müller R, Nielsen TH: Dissecting the plant transcriptome and the regulatory responses to phosphate deprivation. Physiol Plant 2010, 139:129-143.

32. Cheng Y, Zhou W, El Sheery NI, Peters C, Li M, Wang X, Huang J: Characterization of the Arabidopsis glycerophosphodiester phosphodiesterase (GDPD) family reveals a role of the plastid-localized AtGDPD1 in maintaining cellular phosphate homeostasis under phosphate starvation. Plant J 2011, 66:781-795.

33. Russo MA, Quartacci MF, Izzo R, Belligno A, Navari-Izzo F: Long- and short-term phosphate deprivation in bean roots: plasma membrane lipid alterations and transient stimulation of phospholipases. Phytochemistry 2007, 68:1564-1571.

34. Hammond JP, Bennett MJ, Bowen HC, Broadley MR, Eastwood DC, May ST, Rahn C, Swarup R, Woolaway KE, White PJ: Changes in gene expression in Arabidopsis shoots during phosphate starvation and the potential for developing smart plants. Plant Physiol 2003, 132:578-596.

35. Secco D, Jabnoune M, Walker H, Shou H, Wu P, Poirier Y, Whelana J: Spatio-temporal transcript profiling of rice roots and shoots in response to phosphate starvation and recovery. Plant Cell 2013. doi:http://dx.doi.org/10.1105/tpc.113.117325.

36. Bais HP, Prithiviraj B, Jha AK, Ausubel FM, Vivanco JM: Mediation of pathogen resistance by exudation of antimicrobials from roots. Nature 2005, 434:217-221

37. Millet YA, Danna CH, Clay NK, Songnuan W, Simon MD, Werck-Reichhart D, Ausubel FM: Innate immune responses activated in Arabidopsis roots by microbe-associated molecular patterns. Plant Cell 2010, 22:973-990.

38. Muchhal US, Pardo JM, Raghothama KG: Phosphate transporters from the higher plant Arabidopsis thaliana. Proc Natl Acad Sci U S A 1996, 93:10519-10523.

39. Brutnell TP, Wang L, Swartwood K, Goldschmidt A, Jackson D, Zhu XG, Kellogg E, Van Eck J: Setaria viridis: a model for C4 photosynthesis. Plant Cell 2010, 22:2537-2544.

40. Malamy JE, Benfey PN: Organization and cell differentiation in lateral roots of Arabidopsis thaliana. Development 1997, 124:33-44.

doi:10.1186/1471-2229-14-69

Cite this article as: Alatorre-Cobos et al:: An improved, low-cost, hydroponic system for growing Arabidopsis and other plant species under aseptic conditions. BMC Plant Biology 2014 14:69.

\section{Submit your next manuscript to BioMed Central and take full advantage of:}

- Convenient online submission

- Thorough peer review

- No space constraints or color figure charges

- Immediate publication on acceptance

- Inclusion in PubMed, CAS, Scopus and Google Scholar

- Research which is freely available for redistribution 